www. revis tad y o. com

\title{
Identificación de los elementos organizativos clave en el desempeño de las empresas sociales. El caso de los telecentros en Latinoamérica
}

Identifying key organizational elements in the performance of social enterprises. The case of telecentres in Latin America

Ramon Fisac-Garcia, Ana Moreno-Romero, Manuel Acevedo-Ruiz y Jesús Juan-Ruiz

Departamento de Ingeniería de Organización, Administración de Empresas y Estadística. Escuela Técnica Superior de Ingenieros Industriales de Madrid. Universidad Politécnica de Madrid (UPM). c/José Gutiérrez Abascal 2, Madrid, 28023, España.

ramon.fisac@upm.es, ana.moreno.romero@upm.es, manuel@manuelacevedo.net, jesus.juan@upm.es

Fecha de recepción: 15-10-2014

Fecha de aceptación: 10-04-2015

Resumen: Las tecnologías han transformado radicalmente el acceso a muchos servicios en la actualidad (educación, cultura, empleo, etcétera). Los telecentros son empresas sociales que tienen por objetivo proveer acceso a las tecnologías y a la información para un alto porcentaje de la población mundial. En este trabajo, de carácter exploratorio, se estudian los principales aspectos organizativos de estas entidades y se determinan los factores clave en su desempeño. A través de una encuesta se evaluó la precepción de los gestores de 49 telecentros en Latinoamérica sobre los principales aspectos organizativos de sus centros así como de su desempeño.

Palabras clave: empresa social, desempeño, telecentros, elementos clave.

Abstract: Social enterprise is an organizational model based on the sale of products and services to achieve a social mission. Social enterprises are characterized by two elements: first, it pursues a social mission, defined by a societal problem or an environmental challenge. Second, it is uses a market-based approach consisting on the sale of goods and/or services. It represents an interesting alternative for projects aiming to provide access to basic goods and services as it relies on a self-generated income formula and a flexible model which supports economic and social sustainability.

The goal of this article is to analyze the main organizational elements of a sample of telecentres and to explore whether a relationship can be identified between some of the elements and the organization performance. A better knowledge in these issues can help existing organizations improve its sustainability and the design of social enterprises.

This article draws on a previous organizational model for the analysis of social enterprises developed at the Universidad Politecnica de Madrid. The model consists of 8 elements divided into 3 different categories: those referring to the goals of the initiative (what is to be done), those describing the people working on the initiative (stakeholders) and those related to the operational elements.

The empirical study has been carried out within Telecentre.org, an international foundation that supports the establishment and sustainability of grassroots level telecentres. These entities provide access to communication and information services for economic, social and cultural development in local communities. The study consist of a 36-item questionnaire designed and developed by the authors to capture the perceptions of telecentre managers on the most relevant organizational aspects of their organizations, and on four dimensions of the telecentre performance: social, economic, customer satisfaction and employees and volunteer satisfaction.

A factor analysis transformation is done to summarize the information contained in the 36 items of the questionnaire. Then, correlation studies and regressions were carried out to identify the relations among the different variables.

The results show that the global performance is highly correlated with seven of the elements of the organizational analysis model used. Organizational culture emerges as an element that explains a big part of the global performance, the customer satisfaction and the employees/volunteer satisfaction.

Keywords: social enterprise, performance, telecentre, key factors. 


\section{Introducción}

El mundo ha experimentado un desarrollo económico de gran importancia en los últimos años pero, en la actualidad, son muchas las personas que no son capaces de llevar una vida digna y satisfacer sus necesidades básicas. El emprendimiento social contempla diferentes aproximaciones por parte del sector de la sociedad civil a la resolución de estos problemas con estrategias muy diversas, desde visiones reivindicativas y de denuncia hasta el uso de mecanismos de mercado.

Entre estas iniciativas, la empresa social es un modelo organizativo que presenta un interesante potencial para resolver problemáticas sociales (Paton, 2003; Thompson \& Doherty, 2006). El interés por la empresa social ha surgido tanto en países industrializados como en economías en transición y en países en vías de desarrollo (Seelos \& Mair, 2005) porque representa un modelo organizativo alternativo dentro del capitalismo y con gran potencial (Amin, 2009; Hemingway, 2005; Hudson, 2009; Wilson \& Post, 2013). En particular, las características que hacen interesante a la empresa social como herramienta para ayudar a resolver los problemas sociales del mundo son la combinación de un objetivo social que es alcanzado a través de una visión empresarial y su flexibilidad de adaptación a los diferentes contextos del mundo.

Algunos autores (Doherty et al., 2014) destacan la escasez de estudios empíricos que acompañen el desarrollo teórico de la empresa social. Siendo el consenso conceptual el principal caballo de batalla de los últimos años, existen pocas evidencias sobre cómo funcionan las empresas sociales y sobre sus claves de su éxito. La mejora en el estudio aplicado o empírico pasa por la construcción de estas herramientas para que tanto académicos como organizaciones sobre el terreno mejoren su comprensión sobre los mecanismos internos de las empresas sociales.

En este contexto, el objetivo de este artículo es estudiar los principales elementos organizativos observables de una empresa social e identificar aquellos que son clave en su desempeño.
Este estudio empírico tiene un carácter exploratorio y consiste en el análisis de un caso particular de empresa social: los telecentros. Los telecentros son espacios públicos para el acceso y uso efectivo de las tecnologías de la información y la comunicación (TIC), particularmente a Internet, en lugares donde existen dificultades para satisfacer esta necesidad para al menos una parte de la población. La mayoría de estas organizaciones ${ }^{1}$ son auténticas empresas sociales ya que buscan la resolución de una problemática social (la falta de acceso a la información y de oportunidades relacionadas con las TIC) a través de mecanismos de mercado (la venta de productos y servicios). El estudio de estas entidades resulta de gran interés porque proporcionan un servicio fundamental para el desarrollo en el siglo XXI (educación, sanidad, cultura, empleo) y porque se encuentran ampliamente extendidas por todo el mundo, llegando a los rincones más remotos del planeta.

El artículo se estructura como sigue. En el epígrafe 2 se presenta el concepto de empresa social junto con un modelo para el análisis organizativo de estas entidades propuesto por los autores en resultados de investigación previos de esta línea (Fisac et al., 2011). En el epígrafe 3, se analiza el caso de los telecentros como empresa social y se presentan las claves del estudio empírico realizado, para a continuación, en el epígrafe 4, profundizar en la metodología utilizada en el mismo. En el epígrafe 5, se presentan los resultados del tratamiento estadístico de los datos recogidos en este estudio, realizando un análisis de correlaciones entre los elementos organizativos del modelo presentado en el epígrafe 1 y la percepción del despeño de los telecentros, así como una identificación de los elementos organizativos que explican este desempeño a través de regresiones lineales. Finalmente en el epígrafe 6 se presentan conclusiones, limitaciones y futuros desarrollos de esta investigación.

\footnotetext{
${ }^{1}$ En rigor, no se puede decir que todos los telecentros funcionan como empresas sociales o aspiran a ello. Hay redes estatales en las que operan como bibliotecas, es decir, sin intención de generar ingresos.
} 


\section{La empresa social}

\subsection{Concepto}

La empresa social es un concepto que no presenta, ni en la literatura académica ni en la práctica, una definición precisa en la actualidad (Alter, 2007; Doherty et al., 2014; Galera \& Borzaga, 2009; Brouard \& Larivet, 2011; Haugh, 2012), existiendo diversas nociones de este concepto en diferentes países y regiones del mundo (Galera \& Borzaga, 2009).

Debido a la cantidad de enfoques legítimos, la tendencia que se observa en la literatura (Pearce, 2003; Nichols, 2006; Ridley-Duff, 2008; Ridley-Duff \& Bull, 2011; Bull et al., 2010) es reconocer un tipo ideal de empresa social definida por una serie de criterios económicos y sociales que "no representan condiciones que una organización debe cumplir para calificarse como empresa social, sino unos criterios preceptivos" (Defourny \& Nyssens, 2008: 48).

Con el fin de no extender el debate sobre la definición de empresa social, diremos que la empresa social es un concepto en el que caben diferentes interpretaciones y delimitaciones, si bien sus principios fundamentales son comunes (Laville \& Nyssens 2001; Mair \& Martí, 2006; Peredo \& McLean 2006; Peattie \& Morley 2008; Doherty et al., 2014):

- La razón de ser y la misión de la organización está definida por un objetivo social, como la lucha contra la exclusión, la provisión de servicios básicos o la conservación del medio ambiente.

- $\quad$ Esta misión social se persigue mediante la realización de una actividad económica en un mercado competitivo (venta de productos y servicios).

Además de estos criterios, algunos autores (Defourny \& Nyssens, 2012) destacan otros como la existencia de participación democrática o la reinversión de los excedentes generados.

\subsection{Modelo de análisis organizativo}

Para establecer los aspectos organizativos más relevantes de una empresa social, se ha empleado un modelo de análisis desarrollado por el Grupo de Investigación en Organizaciones Sostenibles (GIOS) de la Universidad Politécnica de Madrid (Fisac et al., 2011). Este modelo, que se representa gráficamente en la figura 1, analiza los principales elementos que describen el funcionamiento de una empresa social.

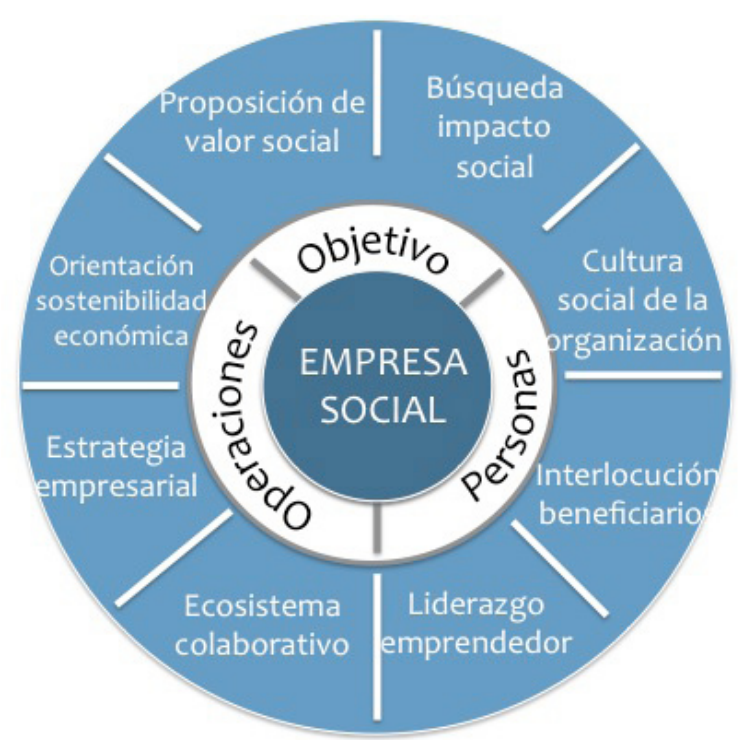

Figura 1. Modelo para el análisis organizativo de empresas sociales

Se identifican ocho elementos agrupados en tres categorías: Objetivos de la organización, Personas y Operaciones. Los elementos representan aspectos organizativos que están presentes en diferentes grados en las empresas sociales y con los que los promotores diseñan la organización y la adaptan a su contexto institucional, social y económico. La tabla 1 explica brevemente los ocho elementos de caracterización de la empresa social. 
Tabla 1. Descripción de elementos del modelo para el análisis organizativo de empresas sociales

\begin{tabular}{|c|c|}
\hline Elemento & Descripción \\
\hline \multicolumn{2}{|c|}{ Elementos relativos a los objetivos de la organización } \\
\hline Proposición de valor social & $\begin{array}{l}\text { Beneficios que la organización crea en su lucha contra la problemática social. Puede incluir el } \\
\text { acceso a productos y servicios, provisión de servicios sociales, empleo y protección de la natu- } \\
\text { raleza, etcétera. } \\
\text { Normalmente esta proposición de valor presenta algún grado de innovación (en el producto/ } \\
\text { servicio, canal o modelo de negocio) y se encuentra adaptado a los beneficiarios. }\end{array}$ \\
\hline Orientación al impacto social & $\begin{array}{l}\text { Compromiso de permanencia con la comunidad y consecución de un impacto social a largo } \\
\text { plazo. El desarrollo social es el objetivo último de la iniciativa y por ello emplea sus recursos y } \\
\text { capacidades para este fin. }\end{array}$ \\
\hline \multicolumn{2}{|l|}{ Elementos relativos a las personas } \\
\hline Cultura social de la organización & $\begin{array}{l}\text { Conjunto de valores compartidos, comportamientos y actitudes que definen una "manera de } \\
\text { hacer" social de la organización y que permea en las operaciones cotidianas de la organización. } \\
\text { Este cultura puede verse reflejada, por ejemplo, en una participación significativa de los benefi- } \\
\text { ciarios en la gestión y control de la organización. }\end{array}$ \\
\hline Conexión con los beneficiarios & $\begin{array}{l}\text { Mecanismos y medios para trasladar una proposición de valor efectiva a los beneficiarios, que } \\
\text { requiere un contacto cercano y directo con el territorio y las personas: un canal de comunicación } \\
\text { efectivo o un medio de distribución físico, entre otros. }\end{array}$ \\
\hline $\begin{array}{l}\text { Liderazgo emprendedor y gober- } \\
\text { nanza }\end{array}$ & $\begin{array}{l}\text { Existe un líder o grupo de líderes que llevan a cabo un doble cometido: la dirección de las activi- } \\
\text { dades y operaciones de la organización y la motivación de los trabajadores hacia la consecución } \\
\text { de los objetivos. }\end{array}$ \\
\hline \multicolumn{2}{|l|}{ Elementos relativos a las operaciones } \\
\hline Ecosistema colaborativo & $\begin{array}{l}\text { Las empresas sociales no suelen trabajar aisladamente sino que se apoyan en diferentes soci- } \\
\text { os o aliados: voluntarios, organizaciones no gubernamentales, entidades públicas y empresas } \\
\text { privadas entre otras. Estos actores constituyen una fuente de recursos (económicos, físicos, } \\
\text { humanos) para llevar a cabo las actividades de la empresa. }\end{array}$ \\
\hline Estrategia empresarial & $\begin{array}{l}\text { Las empresas sociales tienen una estrategia empresarial porque combinan una serie de car- } \\
\text { acterísticas propias de las entidades mercantiles: una actividad principal de venta de bienes y } \\
\text { servicios, la competencia en un mercado por clientes e inversores y la búsqueda de la eficiencia } \\
\text { en los procesos internos. }\end{array}$ \\
\hline $\begin{array}{l}\text { Orientación a la sostenibilidad } \\
\text { económica }\end{array}$ & $\begin{array}{l}\text { Las empresas sociales deben limitar su dependencia económica y financiera de agentes exter- } \\
\text { nos (donaciones, subvenciones). Aunque estos mecanismos sean convenientes o necesarios, } \\
\text { debe existir una orientación hacia poder cubrir los costes operativos con los propios ingresos. }\end{array}$ \\
\hline
\end{tabular}




\section{Aplicación al caso telecentros en Latinoamérica}

\subsection{Los telecentros como empresas sociales generadoras de desarrollo}

Los telecentros pueden ser definidos como "centros públicos de encuentro equipados con tecnologías de la información y la comunicación (TIC) consistentes en ordenadores con acceso a Internet que ofrecen acceso público a servicios de comunicación e información para el desarrollo económico, social y cultural de las comunidades locales" (Rao, 2008:476).

Un telecentro está compuesto por diferentes estaciones de trabajo y cuenta, como mínimo, con la presencia de un gestor que se ocupa de los aspectos técnicos (orientación a usuarios, soporte), de labores de gestión (cobro por servicios, gestión de espacios, etcétera) y de la orientación y formación de usuarios. Los telecentros de mayor tamaño o más sofisticados pueden contar con personal adicional para, por ejemplo, ofrecer formación. Los telecentros se caracterizan por su orientación social ${ }^{2}$ siendo la mayoría de sus usuarios personas de renta baja que no pueden disponer de equipos informáticos propios.

Los servicios ofrecidos por estas entidades son diversos: acceso a Internet, cursos de informática, fotocopias e impresiones, envíos de paquetes o dinero, locutorio telefónico, venta de software y accesorios informáticos, o servicios como el desarrollo de webs y aplicaciones o la reparación de ordenadores.

Los telecentros se consideran auténticas empresas sociales porque cumplen los criterios especificados anteriormente:

- $\quad$ La misión principal de la organización es de carácter social y aunque combinan objetivos de lucro y sociales, su objetivo principal es social: luchar contra la falta de acceso a la información, la comunicación y oportunidades digitales (como fuentes de trabajo y comercio electrónico entre otras). Además, los telecentros son significativos canales generadores de cohesión social y participación ciudadana: economía, empleo, educación, salud y gobierno electrónico.

\begin{abstract}
La organización lleva a cabo su misión mediante una actividad principal que es la venta de productos y/o servicios en un mercado.
\end{abstract}

Además, se puede decir que la mayoría de ellos destinan sus beneficios a su misión social y tratan de limitar su funcionamiento a la financiación externa.

\subsection{Descripción de la muestra estudiada}

Para el desarrollo del estudio empírico se contó con la participación de una fundación internacional que apoya la creación y sostenibilidad de telecentros en todo el mundo. La Fundación Telecentre.org está constituida por más de 300 redes que agrupan unos 100.000 telecentros comunitarios y más de 200.000 personas que tienen una participación directa en la organización.

La selección de las cuatro redes participantes en el estudio empírico(Conexión, ATN, POETA y Soriano), todas ellas en la región latinoamericana, se realizó por un mecanismo de autoselección (Saunders et al., 2009), es decir, se prestaron voluntariamente a ser objeto de estudio aquellas redes que estaban más interesadas en participar en el mismo.

Una vez identificadas las redes voluntarias, los coordinadores de éstas seleccionaron la composición de la muestra guiados por unas indicaciones del equipo investigador para garantizar la representatividad de la misma.

En la selección de la muestra se tuvieron en cuenta los factores clave fuente de la heterogeneidad del universo a estudiar: la nacionalidad del telecentro, su carácter rural o urbano, la madurez de su actividad y su dimensión:

Los centros pertenecen a 11 países pertenecientes a las redes: Brasil, Colombia, Costa Rica, Ecuador, El Salvador, Guatemala, México, Perú, República Dominicana, Uruguay y Venezuela.

Los centros se encuentran situados en núcleos de diferentes tamaños, desde pequeñas localidades de 456 habitantes hasta grandes urbes de más de 8 millones de habitantes.

\footnotetext{
${ }^{2}$ Se excluyen de la categoría de telecentros aquellos espacios de negocios que no tienen una orientación hacia la provisión de acceso a las tecnologías de carácter social, como los business centers.
} 
Los centros tienen su comienzo de operación en momentos temporales diferentes, comprendidos entre 2001 y 2014.

- $\quad$ El tamaño de los telecentros varía entre quioscos con un único puesto de trabajo hasta centros de 121 puestos.

Las principales características del estudio empírico se recogen en la tabla 2.

Tabla 2. Datos clave sobre el estudio empírico

\begin{tabular}{|c|c|}
\hline Objeto de estudio & $\begin{array}{l}\text { Aspectos organizativos clave } \\
\text { y desempeño de los telecen- } \\
\text { tros }\end{array}$ \\
\hline Muestra & $\begin{array}{l}49 \text { telecentros pertenecientes } \\
\text { a } 4 \text { redes (Conexión, ATN, } \\
\text { Soriano y POETA) asociadas } \\
\text { a la Fundación internacional } \\
\text { Telecentre.org. }\end{array}$ \\
\hline Alcance & 11 países latinoamericanos \\
\hline Recogida de datos & Abril-Septiembre 2014 \\
\hline $\begin{array}{l}\text { Modelo teórico sobre el que } \\
\text { se basa el cuestionario }\end{array}$ & Fisac et al., 2011 \\
\hline
\end{tabular}

\section{Metodología}

A partir del modelo de análisis organizativo de empresas sociales (Fisac et al., 2011) se elaboró un cuestionario para identificar la presencia de elementos organizativos característicos de la empresa social en los telecentros de la muestra.

Para cada uno de los ocho elementos del modelo se plantearon 4 ó 5 preguntas relativas a los aspectos organizativos más relevantes de las organizaciones encuestadas. El cuestionario se compone de un total de 36 preguntas que recogen las precepciones del gestor del telecentro mediante una valoración en una escala tipo Likert de 0 a 5 puntos.

Adicionalmente, se solicitó al encuestado la valoración del desempeño de la organización en cuatro dimensiones: desempeño económico (ítem 37.1), desempeño social (ítem 37.2), satisfacción de los clientes/beneficiarios (ítem 37.3) y satisfacción de empleados y trabajadores (ítem 37.4). El cuestionario se recoge en el Anexo I.
Para asegurar que los encuestados entendían el objetivo del estudio empírico y las tareas necesarias a realizar, se ofrecieron 3 seminarios mediante una aplicación web (o webinarios) en los que se presentó el objetivo de la investigación a los participantes y se explicó el procedimiento para rellenar el cuestionario.

Una vez validado y testado, el cuestionario se distribuyó a los gestores de la muestra de 49 telecentros seleccionados. Las respuestas de los gestores de los telecentros fueron tratadas mediante el software informático SPSS.

El análisis de los resultados de la encuesta muestra una alta correlación entre las 40 variables extraídas del cuestionario (correspondientes a las 40 variables estudiadas: 36 elementos organizativos y 4 dimensiones del desempeño). Para simplificar el estudio y extraer la máxima información de los datos se realizó un análisis de componentes principales. El procedimiento seguido fue el siguiente:

- Se comprobó para cada uno de los ocho bloques de preguntas (correspondientes a los ocho elementos del modelo) la correlación positiva y muy significativa entre sus variables (respuestas a cada pregunta).

- Se obtuvo un único indicador combinando linealmente la información de las variables del bloque, de manera que resumiera la información conjunta de dichas variables. Este indicador se obtuvo mediante la técnica de componentes principales.

- Se obtuvo un indicador del desempeño (llamado desempeño global) combinando linealmente las cuatro variables de desempeño del telecentro (social, económico, satisfacción de clientes y satisfacción de empleados/voluntarios).

Con esta transformación se consigue resumir la información de las preguntas correspondientes a cada elemento en un único factor (un total de ocho factores, uno por cada uno de los bloques 1-8) y la información de las cuatro dimensiones del desempeño en una variable de desempeño global. Las nuevas variables y sus características se recogen en la tabla 3 . 
Tabla 3. Nuevas variables obtenidas en la reducción de dimensiones

\begin{tabular}{|c|c|c|c|}
\hline Elemento del modelo & Factor & Preguntas contenidas & Varianza total explicada (\%) \\
\hline Proposición de valor social & Factor_A & $1,2,3,4$ y 5 & 56,2 \\
\hline Búsqueda de impacto social & Factor_B & $6,7,8$ y 9 & 65,43 \\
\hline Cultura de empresa social & Factor_C & $10,11,12,13$ y 14 & 53,30 \\
\hline Interlocución con beneficiarios & Factor_D & $15,16,17$ y 18 & 61,49 \\
\hline Liderazgo emprendedor & Factor_E & $19,20,21$ у 22 & 68,75 \\
\hline Ecosistema colaborativo & Factor_F & $23,24,25$ у 26 & 59,44 \\
\hline Estrategia empresarial & Factor_G & $27,28,29,30$ у 31 & 45,35 \\
\hline Or. sostenibilidad económica & Factor_H & $32,33,34,35$ y 36 & 30,79 \\
\hline Or. sostenibilidad económica & Factor_H & $32,33,34,35$ y 36 & 30,79 \\
\hline $\begin{array}{l}\text { Desempeño social, económico, } \\
\text { satisfacción de clientes, satisfac- } \\
\text { ción de empleados y voluntarios }\end{array}$ & Desempeño global & $37.1,37.2,37.3$ у 37.4 & 56,01 \\
\hline
\end{tabular}

En la última columna se observa que cada uno de los nuevos factores explica un alto porcentaje de información de las preguntas del cuestionario. Siete de las nueve variables generadas tienen un porcentaje de la varianza total explicada superior al $50 \%$, y solamente dos de ellas (las correspondientes a "Estrategia empresarial" y "Orientación a la Sostenibilidad Económica") ofrecen valores menores a este umbral ${ }^{3}$.

Los nuevos indicadores obtenidos permiten identificar los elementos de la organización que mejor explican el desempeño de la misma (es decir, aquellos que tienen mayor correlación). Los modelos utilizados han sido de regresión múltiple y sus resultados se presentan en las secciones siguientes.

\section{Resultados}

\subsection{Análisis descriptivo de los elementos organizativos del modelo y de las percepciones de desempeño}

Cada bloque de preguntas del modelo evalúa el grado de identificación de la organización estudiada con los elementos del modelo de análisis de empresa social.

La tabla 4 muestra los resultados de las puntuaciones de las preguntas integrantes de cada elemento del modelo. La ponderación se realizó otorgando un peso relativo igual para todas las preguntas ${ }^{4}$.

\footnotetext{
${ }^{3}$ Este hecho planteó la posibilidad de extraer dos componentes principales para cada uno de estos dos elementos, pero por simplicidad metodológica se mantuvo una única componente por elemento para el posterior análisis.

${ }^{4}$ Se puede discutir que dentro de cada uno de los elementos del modelo existen preguntas o elementos más importantes, pero en una primera aproximación se estudiaron los resultados con un mismo peso relativo. En posteriores usos del modelo se investigarán otras ponderaciones.
} 
Tabla 4. Resultados medios de la encuesta para los telecentros

\begin{tabular}{|c|c|c|c|}
\hline & Media & $\begin{array}{c}\text { Caso más } \\
\text { desfavorable }\end{array}$ & $\begin{array}{l}\text { Caso más } \\
\text { favorable }\end{array}$ \\
\hline $\begin{array}{l}\text { Proposición de } \\
\text { valor social }\end{array}$ & 4,27 & 2,60 & 4,80 \\
\hline $\begin{array}{l}\text { Búsqueda de } \\
\text { impacto social }\end{array}$ & 4,01 & 0,50 & 5,00 \\
\hline $\begin{array}{l}\text { Cultura de empre- } \\
\text { sa social }\end{array}$ & 3,80 & 2,80 & 5,00 \\
\hline $\begin{array}{l}\text { Interlocución con } \\
\text { los beneficiarios }\end{array}$ & 4,44 & 4,00 & 5,00 \\
\hline $\begin{array}{l}\text { Liderazgo em- } \\
\text { prendedor }\end{array}$ & 4,38 & 3,50 & 5,00 \\
\hline $\begin{array}{l}\text { Ecosistema colab- } \\
\text { orativo }\end{array}$ & 4,10 & 2,00 & 5,00 \\
\hline $\begin{array}{l}\text { Estrategia empre- } \\
\text { sarial }\end{array}$ & 3,73 & 1,60 & 4,80 \\
\hline $\begin{array}{l}\text { Orientación a la } \\
\text { sostenibilidad } \\
\text { económica }\end{array}$ & 3,30 & 2,80 & 3,60 \\
\hline Media & 4,00 & 2,48 & 4,78 \\
\hline
\end{tabular}

Estos mismos valores se representan gráficamente en la figura 2, que muestra las puntuaciones para los 8 bloques en un gráfico de radar.

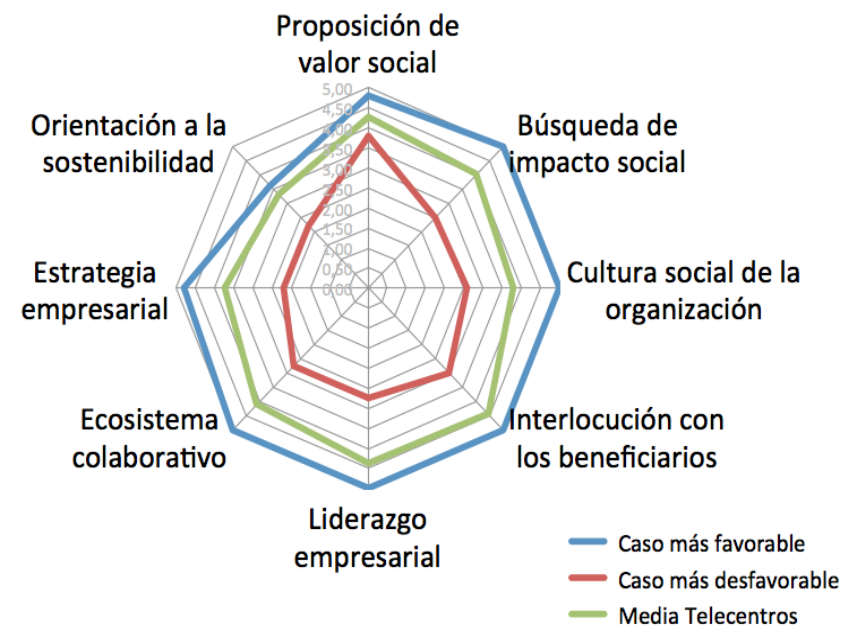

Figura 2. Resultados en la valoración de los elementos del modelo

La tabla 5 muestra los resultados del análisis de las percepciones de los gestores de los centros sobre los aspectos de desempeño considerados

\begin{tabular}{|c|c|}
\hline Elemento & Media \\
\hline Desempeño social & 4,31 \\
\hline Desempeño económico & 3,19 \\
\hline Satisfacción clientes & 4,37 \\
\hline $\begin{array}{c}\text { Satisfacción empelados } \\
\text { y voluntarios }\end{array}$ & 4,21 \\
\hline
\end{tabular}

Se observan valores parecidos para todos los desempeños (en torno a 4,3 puntos), excepto para el desempeño económico, donde el valor es sensiblemente menor $(3,19)$.

\subsection{Análisis de las correlaciones entre los elementos organizativos del modelo, las percepciones de desempeño y sus dimensiones}

La tabla 6 muestra los resultados del análisis de correlaciones entre los elementos del modelo para los telecentros encuestados.

Tabla 6. Correlación entre los elementos del modelo y el desempeño global

\begin{tabular}{|c|c|c|}
\hline $\begin{array}{c}\text { Elemento del } \\
\text { modelo }\end{array}$ & Factor & $\begin{array}{c}\text { Correlación con } \\
\text { Desempeño global }\end{array}$ \\
\hline $\begin{array}{c}\text { Proposición de valor } \\
\text { social }\end{array}$ & Factor_A & $0,739^{* *}$ \\
\hline $\begin{array}{c}\text { Búsqueda de impac- } \\
\text { to social }\end{array}$ & Factor_B & $0,689^{* *}$ \\
\hline $\begin{array}{c}\text { Cultura de empresa } \\
\text { social }\end{array}$ & Factor_C & $0,732^{* *}$ \\
\hline $\begin{array}{c}\text { Interlocución con } \\
\text { beneficiarios }\end{array}$ & Factor_D & $0,634^{* *}$ \\
\hline $\begin{array}{c}\text { Liderazgo em- } \\
\text { prendedor }\end{array}$ & Factor_E & $0,584^{* *}$ \\
\hline $\begin{array}{c}\text { Ecosistema colabo- } \\
\text { rativo }\end{array}$ & Factor_F & $0,475^{* *}$ \\
\hline $\begin{array}{c}\text { Estrategia empre- } \\
\text { sarial }\end{array}$ & Factor_G \\
\hline $\begin{array}{c}\text { sostenibilidad } \\
\text { económica }\end{array}$ & Factor_H & $0,542^{* *}$ \\
\hline
\end{tabular}

(Se muestra en la tabla los valores para la Correlación de Pearson. * $p$-valor indica menor que $0,05,{ }^{* *}$ indica $p$-valor menor que 0,01 ) 
El estudio de las correlaciones entre estas nuevas variables permitió observar que todos los elementos del modelo, salvo el factor $\mathrm{H}$ (orientación a la sostenibilidad económica) están altamente correlacionados con el desempeño global. Los 7 elementos presentan correlación positiva y muy significativa (nivel de significación $=0,01$ ), lo que puede querer decir que son con- ceptos que influyen de manera positiva en el desempeño del telecentro. Es decir, un incremento en el nivel de cualquiera de los 8 elementos está relacionado con un incremento positivo del desempeño global. La tabla 7 muestra los resultados del análisis de correlaciones de las percepciones de desempeño de los gestores de telecentros encuestados.

Tabla 7. Correlaciones entre las dimensiones del desempeño

\begin{tabular}{|c|c|c|c|c|}
\hline Componente & Desempeño social & $\begin{array}{l}\text { Desempeño } \\
\text { económico }\end{array}$ & Satisfacción clientes & $\begin{array}{c}\text { Satisfacción empleados y } \\
\text { voluntarios }\end{array}$ \\
\hline Desempeño social & 1 & 0,096 & $0,505^{\star \star}$ & $0,474^{* *}$ \\
\hline Desempeño económico & & 1 & 0,221 & 0,215 \\
\hline Satisfacción de clientes & & & 1 & $0,738^{* *}$ \\
\hline $\begin{array}{l}\text { Satisfacción de empleados } \\
\text { y voluntarios }\end{array}$ & & & & 1 \\
\hline
\end{tabular}

(Se muestra en la tabla los valores para la Correlación de Pearson. *p-valor indica menor que $0,05,{ }^{* *}$ indica p-valor menor que 0,01 )

Del estudio de correlación entre las diferentes dimensiones del desempeño (tabla 7) se desprenden las siguientes observaciones:

El desempeño social, la satisfacción de los clientes y la satisfacción de voluntarios y empleados están altamente correlacionadas. Esto quiere decir que un nivel de desempeño favorable en una de estas dimensiones tiene un efecto positivo sobre las demás.

El desempeño económico no tiene una correlación significativa con ninguna de las demás dimensiones.

La satisfacción de los clientes y la satisfacción de empleados y voluntarios presenta la correlación más alta, con un valor de 0,738 .

\subsection{Identificación de factores organizativos clave del desempeño en los telecentros}

Se realizaron diferentes regresiones lineales para explicar los diferentes desempeños con los elementos del modelo. En la tabla 8 se muestra la combinación de elementos que mejor explica el desempeño y cada una de las dimensiones del desempeño según las regresiones lineales realizadas ${ }^{5}$.

\footnotetext{
${ }^{5}$ Para el desempeño global y para cada una de sus cuatro componentes se realizaron regresiones lineales "forward" y "backward" y se seleccionaron los modelos que mejor explicaban las variables dependientes, es decir, aquellas con mayor R cuadrado.
} 
Tabla 8. Regresiones lineales realizadas para explicar el desempeño global y sus dimensiones

\begin{tabular}{|l|l|c|}
\hline Variable dependiente explicada & Variables independientes obtenidas & R cuadrado \\
\hline Desempeño global & Cultura organizativa, Estrategia empresarial & $64,5 \%$ \\
\hline Desempeño social & Proposición de valor social & $44,2 \%$ \\
\hline Desempeño económico & $\begin{array}{l}\text { Búsqueda de impacto social, Cultura or- } \\
\text { ganizativa, Orientación a la sostenibilidad } \\
\text { económica }\end{array}$ & $36,5 \%$ \\
\hline Satisfacción clientes & $\begin{array}{l}\text { Búsqueda de impacto social, Cultura organi- } \\
\text { zativa, Liderazgo emprendedor }\end{array}$ & $77,1 \%$ \\
\hline Satisfacción empleados y voluntarios & $\begin{array}{l}\text { Búsqueda de impacto social, Cultura organ- } \\
\text { izativa }\end{array}$ & $66,6 \%$ \\
\hline
\end{tabular}

Se observa que la cultura organizativa tiene un papel fundamental para explicar el desempeño global, ya que explica el grado de satisfacción de los clientes, empleados y voluntarios.

De manera similar, la proposición de valor social que ofrecen los telecentros es el principal elemento que parece explicar el desempeño social. En la medida en la que los servicios y productos que ofrece el telecentro se adapten a los clientes y usuarios, se consigue un mayor desempeño social. Sin embargo, el bajo valor de la $\mathrm{R}$ cuadrado sugiere ampliar el estudio de esta componente del desempeño.

Por su parte, el liderazgo emprendedor tiene un papel importante en la satisfacción de los clientes, según los gestores de los telecentros. Una organización que presenta un fuerte liderazgo hacia la comunidad parece satisfacer más a los clientes, en opinión de los gestores de los telecentros.

\section{Conclusiones}

Esta investigación ofrece una contribución al estudio organizativo de los telecentros y avanza algunas líneas de estudio importantes en el campo de la empresa social. El artículo presenta la aplicación de una herramienta para el análisis organizativo de las empresas sociales a una muestra de telecentros en Latinoamérica. Para una muestra de 49 telecentros de distinta condición se evaluó la percepción de sus gestores sobre los principales aspectos organizativos de sus centros así como de su desempeño en cuatro dimensiones: social, económica, satisfacción de clientes y satisfacción de trabajadores.
De los resultados del estudio se pueden obtener relaciones interesantes entre aspectos organizativos y el desempeño de los telecentros. Del estudio de las correlaciones, podemos concluir que existe una alta correlación entre el desempeño y siete de los ocho elementos organizativos del modelo: la proposición de valor social, la búsqueda del impacto social, la cultura organizativa, el liderazgo emprendedor, la conexión con los beneficiarios, el ecosistema colaborativo y la estrategia empresarial.

En particular, se destaca el papel de la cultura organizativa que, en este modelo, explica por si misma el $56 \%$ del desempeño global de la organización y el $60,2 \%$ de la satisfacción de los empleados. Esto significa que la cultura organizativa es un buen indicador del desempeño global de un telecentro.

Del análisis de los resultados se desataca también que el desempeño económico es el principal reto en el estudio de los telecentros y la dimensión en la que éstos son más vulnerables ya que su valoración media, 3,19 puntos, es la más baja de las dimensiones del desempeño. El desafío por la sostenibilidad económica que se desprende de los resultados es coherente con el interés de los telecentros por explorar el modelo de empresa social.

Este hecho junto con otros resultados del estudio (como la falta de correlación significativa del desempeño económico con el desempeño global o con sus demás dimensiones) sugiere que se continúen estudiando los telecentros desde el prisma de la empresa social. 
El análisis empírico puede ayudar a diferentes tareas en la comprensión del funcionamiento y el diseño de empresas sociales. Además, la medida del desempeño contribuye a una mejor comunicación externa y reporte que pueden incrementar las oportunidades para estas organizaciones (financiación, captación de recursos y socios) y mejorar su visibilidad en la sociedad.

Este estudio constituye un paso exploratorio en el estudio empírico de las empresas sociales en el que se ha analizado un tipo específico de ellas (los telecentros) y que, por lo tanto, no permite extender las conclusiones alcanzadas a todo el universo de aquellas. En el futuro, se buscará profundizar en el estudio de los telecentros y aplicar el modelo a otros tipos de empresas sociales, de manera que mejore la comprensión de los aspectos organizativos de este modelo.

\section{Referencias}

ALTER, K. (2007). «Social Enterprise Typology», Virtue Venture, http://www.virtueventures.com/setypology.pdf.

AMIN, A. (2009). «Extraordinarily ordinary: working in the social economy». Social Enterprise Journal, 5 (1), pp.30-49.

BROUARD, F. y LARIVET, S., (2011). «Essay of clarifications and definitions of the related concepts of social enterprise, social entrepreneurs and social entrepreneurship». En Fayolle, A. y Matlay, H. (eds.): Handbook of research on social entrepreneurship, Cheltenham, UK: Edward Elgar Publishing Ltd, pp. 29-56.

BULL, M., RIDLEY-DUFF, R.J., FOSTER, D., y SEANOR, P. (2010). «Conceptualising Ethical Capital in Social Enterprises». Social Enterprise Journal, 6(3). pp. 250-264.

DEFOURNY, J. y NYSSENS, M. (2006). «Defining social enterprise». En Nyssens, M. (ed.): Social Enterprise: at the Crossroads of Market, Public Policies and Civil Society, London: Routledge, pp. 3-26.

DEFOURNY, J. y NYSSENS, M. (2012). «The EMES approach of social enterprise in a comparative perspective». Working paper No. 12/03, EMES European Research Network.

DOHERTY, B., HAUGH, H. y LYON, F. (2014). «Social
Enterprises as Hybrid Organizations: A Review and Research Agenda». International Journal of Management Reviews, 16 (4), pp. 417-436

FISAC, R., MORENO, A., MATAIX, C. y PALACIOS, M. (2011). «La empresa social: revisión de conceptos y modelo para el análisis organizativo». Revista Española del Tercer Sector, 17, pp. 41- 66.

GALERA, G. y BORZAGA, C. (2009). «Social enterprise. An international overview of its conceptual evolution and legal implementation». Social Enterprise Journal, 5 (3), pp. 210-228.

HAUGH, H. (2012). "The importance of theory in social enterprise research». Social Enterprise Journal, 8 (1), pp.7-15.

HEMINGWAY, C.A. (2005). «Personal values as a catalyst for corporate social entrepreneurship». Journal of Business Ethics, 60 (3), pp. 33-249.

HUDSON, R. (2009). "Life on the edge: navigating the competitive tensions between the 'social' and the 'economic' in the social economy and in its relations to the mainstream». Journal of economic geography, 9 (4), pp. 493-510.

LAVILLE, J.L. y NYSSENS, M. (2001). «The social enterprise: towards a theoretical socio-economic approach». En Borzaga, C. y Defourny, J. (Eds): The Emergence of Social Enterprise, London: Routledge, pp. 312-332.

MAIR, J. y MARTÍ, I. (2006). «Social Entrepreneurship research: a source of explanation, prediction, and delight». Journal of World Business, 41 (1), pp. 36-44.

NICHOLS, A. (2006). Social entrepreneurship: New models of sustainable change, Oxford: Oxford University Press.

PATON, R. (2003). Managing and measuring social enterprises, Thousand Oaks: Sage Publications.

PEARCE J. (2003). Social Enterprise in Anytown, London: Calouste Gulbenkian Foundation.

PEREDO, A.M. y McLEAN, M. (2006). «Social entrepreneurship: a critical review of the concept». Journal of World Business, 41 (1), pp. 56-65. 
PEATTIE, K. y MORLEY, A. (2008). «Eight paradoxes of the social enterprise research agenda». Social Enterprise Journal, 4 (2), pp.91-107.

RAO, S.S. (2008). «Social development in Indian rural communities: Adoption of telecentres». International Journal of Information Management, 28 (6), pp. 474-482.

RIDLEY-DUFF, R. (2008). «Social enterprise as a socially rational business». International Journal of Entrepreneurial Behaviour and Research, 14 (5), pp. 291-312.

RIDLEY-DUFF, R.J. y BULL, M. (2011). Understanding Social Enterprise: Theory and Practice, London: Sage Publications.
SAUNDERS, M., LEWIS, P. y THORNHILL, A. (2009). Research methods for business students, Essex: Pearson Education Limited.

SEELOS, C. y MAIR, J. (2005). «Social entrepreneurship: creating new business models to serve the poor», Business Horizons, 48 (3), pp. 241-6.

THOMPSON, J.L. y DOHERTY, R. (2006). "The diverse world of social enterprise: a collection of eight social enterprise stories». International Journal of Social Economics, 33 (5), pp. 361-375

WILSON, F. y POST, J.E. (2013). «Business models for people, planet (\& profits): exploring the phenomena of social business, a market-based approach to social value creation». Small Business Economics, 40 (3), pp. 715-737.

\section{Anexo l: Preguntas del cuestionario y resultados medios obtenidos}

\begin{tabular}{|c|c|c|c|}
\hline & Pregunta & Media & Desviación típica \\
\hline $\mathrm{A} 1$ & $\begin{array}{l}\text { Los productos o servicios que mi telecentro ofrece con- } \\
\text { tribuyen a resolver un problema social o a proveer acceso } \\
\text { a un bien básico }\end{array}$ & 4,43 & 0,707 \\
\hline A2 & $\begin{array}{l}\text { Mi telecentro ofrece productos o servicios adaptados a la } \\
\text { comunidad a la que se dirige }\end{array}$ & 4,41 & 0,804 \\
\hline A3 & Nuestros productos y/o servicios ofrecidos son innovadores & 3,94 & 1,060 \\
\hline A4 & $\begin{array}{l}\text { La solución que proponemos podría ser replicada en otros } \\
\qquad \text { contextos y lugares diferentes }\end{array}$ & 4,43 & 0,736 \\
\hline A5 & $\begin{array}{l}\text { La solución que proponemos es compatible y complemen- } \\
\text { taria con los esfuerzos hechos hasta ahora para solucionar } \\
\text { el problema social }\end{array}$ & 4,15 & 0,945 \\
\hline B6 & $\begin{array}{l}\text { La organización tiene unos objetivos sociales concretos y } \\
\text { explícitamente definidos }\end{array}$ & 4,39 & 1,057 \\
\hline B7 & $\begin{array}{l}\text { El telecentro se dedica de manera exclusiva (o prioritaria) a } \\
\text { resolver el problema social }\end{array}$ & 3,94 & 1,107 \\
\hline B8 & $\begin{array}{l}\text { La organización está comprometida con el cambio social a } \\
\qquad \text { largo plazo }\end{array}$ & 4,18 & 1,074 \\
\hline B9 & $\begin{array}{l}\text { El telecentro realiza actividades periódicas de evaluación de } \\
\text { impacto social para asegurar que su actuación es efectiva }\end{array}$ & 3,53 & 1,427 \\
\hline
\end{tabular}




\begin{tabular}{|c|c|c|c|}
\hline C10 & $\begin{array}{l}\text { Existen formas de vigilar que la misión social de la organ- } \\
\text { ización es prioritaria a la económica }\end{array}$ & 3,92 & 1,182 \\
\hline C11 & $\begin{array}{l}\text { Si existe, la distribución de beneficios económicos en la or- } \\
\text { ganización es limitada }\end{array}$ & 3,38 & 1,290 \\
\hline C12 & $\begin{array}{l}\text { Existen unos valores sociales compartidos que dan forma } \\
\text { a la empresa }\end{array}$ & 4,13 & 0,875 \\
\hline $\mathrm{C} 13$ & $\begin{array}{l}\text { Existen documentos o herramientas que aseguran el com- } \\
\text { portamiento ético de la organización en su conjunto y de sus } \\
\text { integrantes }\end{array}$ & 3,96 & 1,318 \\
\hline C14 & $\begin{array}{c}\text { Existen mecanismos por los que empleados y beneficiarios } \\
\text { pueden influir en la gestión o participar en la propiedad del } \\
\text { telecentro }\end{array}$ & 3,59 & 1,204 \\
\hline D15 & $\begin{array}{l}\text { El telecentro ofrece facilidad de acceso por su horario y lo- } \\
\text { calización }\end{array}$ & 4,51 & 0,845 \\
\hline D16 & $\begin{array}{l}\text { El telecentro se comunica de manera efectiva con la comu- } \\
\text { nidad beneficiaria }\end{array}$ & 4,37 & 0,782 \\
\hline D17 & $\begin{array}{l}\text { La organización tiene buen conocimiento del contexto y la } \\
\text { cultura local }\end{array}$ & 4,45 & 0,679 \\
\hline D18 & $\begin{array}{l}\text { La organización está legitimada y es reconocida por la po- } \\
\text { blación }\end{array}$ & 4,43 & 0,816 \\
\hline E19 & $\begin{array}{l}\text { El telecentro tiene un líder o equipo de líderes que guía la } \\
\text { iniciativa }\end{array}$ & 4,50 & 0,652 \\
\hline E20 & $\begin{array}{l}\text { La visión del líder (o equipo de líderes) es comunicada y } \\
\text { compartida por todos los miembros }\end{array}$ & 4,31 & 0,879 \\
\hline E21 & $\begin{array}{l}\text { El líder (o equipo de líderes) tiene total dedicación y com- } \\
\text { promiso a la iniciativa y está comprometido con la resolución } \\
\text { del problema social }\end{array}$ & 4,37 & 0,859 \\
\hline E22 & Los empleados están comprometidos con el problema social & 4,33 & 0,826 \\
\hline F23 & $\begin{array}{l}\text { Existen acuerdos y colaboraciones habituales con individu- } \\
\text { os y entidades externas como fuente de recursos o de valor }\end{array}$ & 3,96 & 1,398 \\
\hline F24 & $\begin{array}{l}\text { Se utilizan prioritariamente recursos locales (tanto humanos } \\
\text { como físicos) para el funcionamiento de la organización }\end{array}$ & 4,23 & 0,973 \\
\hline F25 & $\begin{array}{l}\text { Si existen, colabora con entidades similares que trabajan } \\
\text { para resolver el mismo problema social }\end{array}$ & 4,13 & 1,142 \\
\hline F26 & $\begin{array}{l}\text { Las actividades colaborativas (o intercambios no monetari- } \\
\text { os) tienen un papel importante para poder cumplir los obje- } \\
\text { tivos del telecentro }\end{array}$ & 4,09 & 1,132 \\
\hline
\end{tabular}




\begin{tabular}{|c|c|c|c|}
\hline G27 & $\begin{array}{l}\text { La principal fuente de ingresos de la organización es la } \\
\text { venta de productos y servicios }\end{array}$ & 3,23 & 1,870 \\
\hline G28 & Existe una estructura organizativa formal & 4,49 & 0,843 \\
\hline G29 & $\begin{array}{l}\text { Una parte significativa de los recursos humanos de la enti- } \\
\text { dad está contratada (es decir, no son voluntarios sino asal- } \\
\text { ariados) }\end{array}$ & 4,17 & 1,260 \\
\hline G30 & $\begin{array}{l}\text { La organización compite en el mercado por las ventas y la } \\
\qquad \text { inversión }\end{array}$ & 2,72 & 1,919 \\
\hline G31 & $\begin{array}{l}\text { La organización cuenta con empleados con capacidades de } \\
\text { gestión empresarial que la dirijan. }\end{array}$ & 4,04 & 1,271 \\
\hline H32 & $\begin{array}{l}\text { La venta de productos y servicios de la empresa producen } \\
\text { unos ingresos suficientes para cubrir los costes operativos. }\end{array}$ & 2,42 & 1,671 \\
\hline $\mathrm{H} 33$ & $\begin{array}{l}\text { La organización tiene una dependencia limitada de fuentes } \\
\text { de ayuda externas: subvenciones, donaciones, etcétera. }\end{array}$ & 3,02 & 1,620 \\
\hline $\mathrm{H} 34$ & $\begin{array}{l}\text { El telecentro adapta su oferta de producto y servicios al pod- } \\
\text { er adquisitivo de los usuarios }\end{array}$ & 4,12 & 1,137 \\
\hline H35 & $\begin{array}{l}\text { El telecentro se encuentra en un ambiente de sostenibilidad } \\
\text { social y política }\end{array}$ & 3,98 & 1,176 \\
\hline $\mathrm{H} 36$ & $\begin{array}{l}\text { La organización tiene acceso a fuentes de aprovisionamien- } \\
\text { to (financiero y de otros recursos) en el mercado }\end{array}$ & 2,98 & 1,625 \\
\hline 37.1 & Desempeño social & 4,31 & 0,822 \\
\hline 37.2 & Desempeño económico & 3,19 & 1,394 \\
\hline 37.3 & Satisfacción clientes & 4,37 & 0,727 \\
\hline 37.4 & Satisfacción empleados y voluntarios & 4,21 & 0,977 \\
\hline
\end{tabular}

